\title{
Distinct generation of subjective vividness and confidence during naturalistic memory retrieval in angular gyrus
}

\author{
Futing Zou' ${ }^{1,5}$ \& Sze Chai Kwok ${ }^{1,2,3,4 *}$
}

${ }^{1}$ Shanghai Key Laboratory of Brain Functional Genomics, Key Laboratory of Brain Functional Genomics Ministry of Education, Shanghai Key Laboratory of Magnetic Resonance, Affiliated Mental Health Center (ECNU), School of Psychology and Cognitive Science, East China Normal University, Shanghai, China

${ }^{2}$ Division of Natural and Applied Sciences, Duke Kunshan University, Kunshan, Jiangsu, China ${ }^{3}$ NYU-ECNU Institute of Brain and Cognitive Science at NYU Shanghai, Shanghai, China ${ }^{4}$ Shanghai Changning Mental Health Center

${ }^{5}$ Department of Psychology, University of Oregon, Eugene, OR, 97403, USA

*Correspondence: sze-chai.kwok@st-hughs.oxon.org

The authors declare that no competing interests exist. 


\begin{abstract}
Subjective experience of remembering is a hallmark of episodic memory, which enables us to monitor experiences, identify mistakes, and adjust our decisions accordingly. A fundamental and enduring puzzle is the origin of confidence in memory; for example, does the confidence during episodic retrieval depend upon the subjective sensed vividness, or does confidence and vividness reflect dissociable introspective processes? The angular gyrus (AnG) exhibits a sensitivity to subjective experience of remembering, but its direct contribution to the monitoring of internal subjective mnemonic experience has hitherto been lacking. Here we combined a novel naturalistic video-watching paradigm with repetitive transcranial magnetic stimulation (rTMS) and restingstate functional magnetic resonance imaging (fMRI) to test the idea that vividness and confidence are generated differently during retrieval. We found that pre-retrieval rTMS targeting the left AnG selectively alters the vividness efficiency compared to control stimulation while keeping metacognitive efficiency and objective memory accuracy unaffected. Using trial-wise data, we showed that vividness mediates the association between confidence and objective memory accuracy and such mediation was eradicated by AnG stimulation. Furthermore, resting-state functional connectivity of hippocampus and AnG was specifically associated with vividness efficiency, while the connectivity of hippocampus and medial prefrontal cortex was associated with metacognitive efficiency across individuals. These findings identify a role for the AnG in gauging the vividness, but not the confidence, of memory, thereby providing evidence for a differentiation account of conscious assessment of memory by functionally and anatomically dissociating the monitoring of vividness from confidence.
\end{abstract}




\section{Introduction}

According to Endel Tulving (Tulving, 1985, 1972), the conception of episodic memory is identified with autonoetic awareness, which gives rise to remembering of personally experienced events. The process of explicitly remembering a specific previous event is often accompanied by a subjective sense of recollection, that is, the subjective vividness of the memory and confidence in the accuracy of the memory. The angular gyrus is widely thought to play a critical role in the integration of mnemonic features into a conscious representation that enables the subjective experience of remembering (Humphreys et al., 2021). For example, fMRI evidence indicates that activity in angular gyrus is associated with subjective reports of vividness (Bonnici et al., 2016; Kuhl and Chun, 2014) and confidence (Qin et al., 2011) during memory retrieval. Consistent with the neuroimaging studies, disruption of left angular gyrus processing by transcranial magnetic stimulation (TMS) selectively reduces confidence but leaving objective recollection accuracy intact (Wynn et al., 2018; Yazar et al., 2014). Although these studies have implicated the angular gyrus in the subjective experience of remembering, the critical role of this region and its functional connectivity with the medial temporal lobe supporting our ability to faithfully monitor internal subjective sense of remembering is not fully understood. Specifically, it remains unclear how important the angular gyrus is involved in the operation of using subjective mnemonic experience to track objective memory performances.

The capacity to evaluate the success of other cognitive processes is known as metacognition (Janet Metcalfe, 1997). Effective metacognition is important for learning and adaptive behavior especially when external feedback is absent, which is often encountered in daily life. A widely used approach to measure this ability lies in the application of signal detection theory or hierarchical Bayes to estimate the sensitivity of self-reported confidence to objective performance (Fleming and Lau, 2014). In human studies of metacognition, there is an increasing amount of work pointing to a network of prefrontal and parietal regions that support and modulate confidence during perceptual decisions (Allen et al., 2017; Morales et al., 2018; Shekhar and Rahnev, 2018). However, the counterpart of metacognition for memory is relatively under-explored. A fundamental and intriguing question is to address the kind of information that is used for generating the confidence rating in memory judgement. Intuitively, one idea is that confidence in memory depends upon the subjective perceived vividness. However, emerging evidence from the perceptual domain suggests that confidence reflects an integration of both internal and external sources of uncertainty (Allen et al., 2016; Bang et al., 2019; Maniscalco and Lau, 2016). On this basis, we reasoned that the computation of vividness and confidence should operate differently during remembering. In this sense, vividness and confidence of memory would be differentially mediated by distinct neural mechanisms and even in different brain regions. We aimed to experimentally verify these dissociable monitoring processes during subjective mnemonic experiences. 
In the present study, we used a novel video-watching paradigm with naturalistic stimuli, in which we aimed to disentangle subjective vividness from confidence during memory retrieval to elucidate whether the vividness and confidence during episodic retrieval reflect dissociable introspective processes. Critically, motivated by evidence for the involvement of angular gyrus in subjective aspects of recollection, we used a combined TMS and MRI approach to test the contribution of angular gyrus to metacognitive and vividness efficiency. To interfere with angular gyrus function, we administered inhibitory $1-\mathrm{Hz}$ repetitive TMS to the left angular gyrus and a control site in a within-subjects design. Moreover, given the known involvement of Brodmann area (BA 10), and more specifically the medial anterior prefrontal cortex (aPFC) and hippocampus in the assessment of subjective memory quality (Baird et al., 2013; Chua et al., 2009; Ford and Kensinger, 2016; Gilboa et al., 2004), we also employed a functional connectivity approach to assess the relationship between functional architecture of these regions and both subjective evaluation abilities.

For an accurate comparison between these subjective experiences we made use of an established concept (Fleming and Daw, 2016; Maniscalco and Lau, 2012). We first recorded memory vividness and confidence judgment under the same objective memory judgment. We then quantified the efficiencies of the two subjective mnemonic judgments by quantifying the trialby-trial correspondence between objective performance and subjective confidence. As the correspondence between objective memory accuracy and subjective confidence increases (i.e., when low confidence ratings follow incorrect trials, and high confidence ratings follow correct trials), metacognitive performance approaches ideal. Equivalently, we further quantified the correspondence between vividness and objective memory performance using an index termed vividness efficiency (vivid-d'/d').

Our design enabled us to test several predictions. First, we predicted that angular gyrus is implicated in the monitoring of vividness but less so in task performance and metacognitive efficiency. That is, the inhibitory TMS to angular gyrus would selectively alter the vividness efficiency while keeping metacognitive efficiency and objective memory accuracy unaffected. Second, on the basis that confidence and objective memory accuracy are intricately linked, we further predicted that the vividness mediates the association between objective memory performance and confidence. Lastly, we predicted the functional connectivity between medial aPFC and hippocampus would be more strongly related to confidence-based metacognition whereas that between angular gyrus and hippocampus more strongly related to vividness efficiency.

\section{Results}




\section{Vividness efficiency is casually dependent on angular gyrus}

While it is often assumed that both vividness ratings and confidence ratings during retrieval mediate subjective experience of remembering, our primary aim was to tease apart these two components of subjective mnemonic experience during retrieval. We operationalized this idea by developing a paradigm, in which participants watched movies at encoding and performed a memory test immediately after receiving TMS inhibition to the angular gyrus (AnG) (Figure 1, see Materials and Methods for details). In the memory test, participants mentally replayed relevant scenes with image cues and rated the vividness of their memory. They also made temporal memory judgments related to the image cues and rated the confidence of their memory responses. The novel and critical manipulation in our experiment is that subjective evaluation efficiency computed by vividness rating and confidence rating are differentiable under the same objective memory judgment.

A

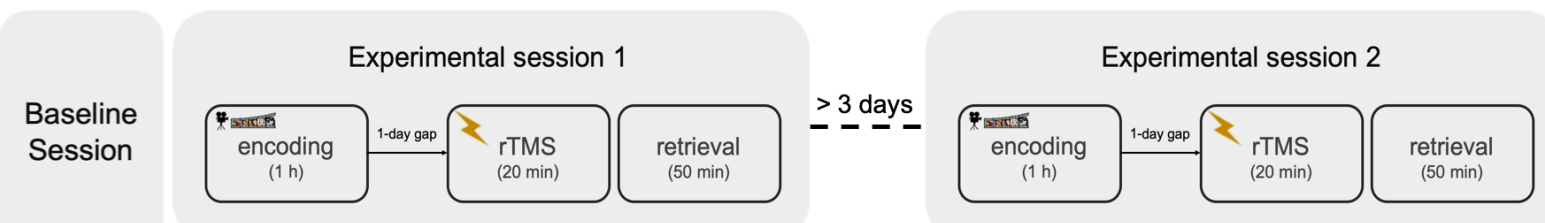

B

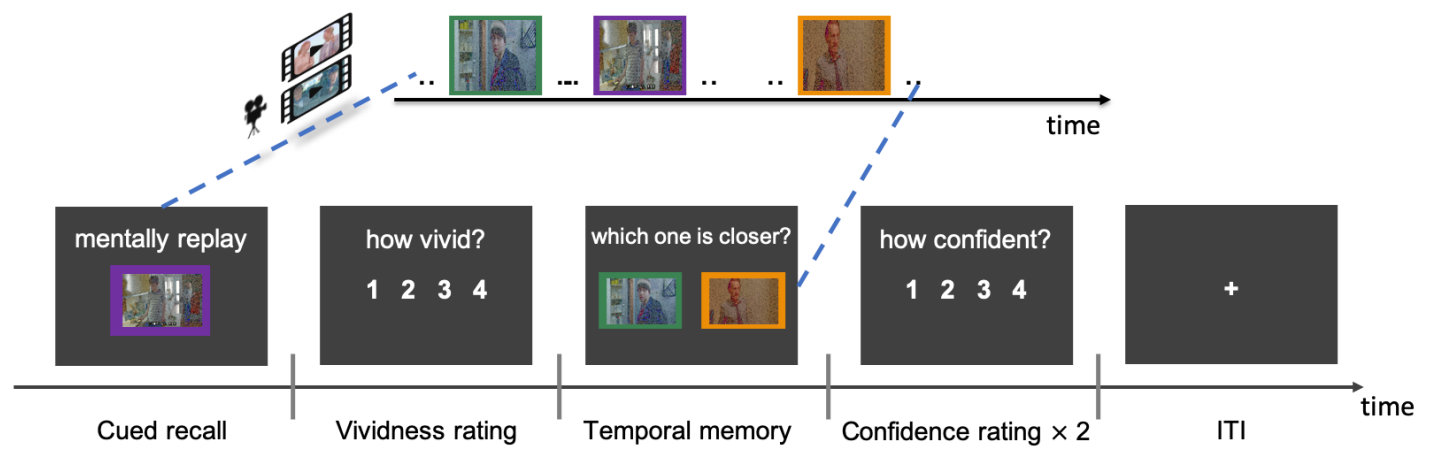

$(6 s)$

(3s)

C

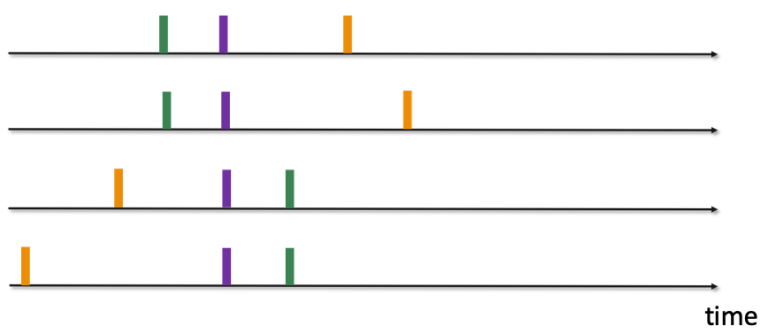

D

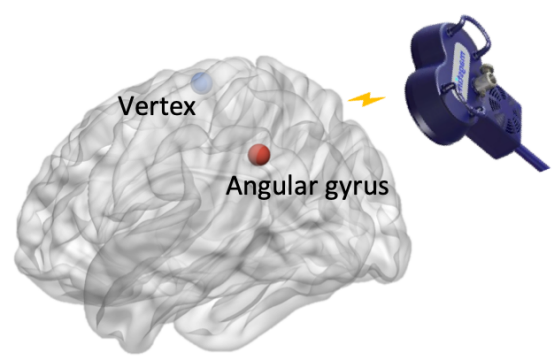

Figure 1. Experimental design. (A) Overview of task design. In each of the experimental sessions, participants viewed a 1-hr movie from Black Mirror at encoding. On the following day, participants received stimulation (over angular gyrus or vertex) and performed the memory test. 
Movie and stimulation sites were assigned in a randomized and counterbalanced order. (B) Schematic overview of the memory test. Trial example: participants mentally replay related scenarios while viewing an image cue from the movie and rated the vividness of their memory. Participants were then presented with another two still frames from the movies and tested on their temporal memory associated with the cue scene, followed by a confidence rating. Each cued recall was followed by two temporal memory judgments. Movie stills in the figure are blurred for copyright reasons. (C) Triad of movie stills selection criteria (purple: cue; green: the closer one to cue; orange: the further one to cue). (D) Stimulation sites: angular gyrus (red, MNI coordinate: $x$ $=-43, y=-66, z=38$ ) and vertex (blue, as control site).

If the self-monitoring of vividness and confidence are dissociable, the effect of AnG stimulation will produce differential effects on the qualities of subjective reports. We first examined the effect of TMS to AnG on basic performance. As expected, TMS did not influence objective memory performance as measured by memory sensitivity $d^{\prime}\left(t_{19}=1.39, p=0.18\right.$; Figure $\left.2 A\right)$ and reaction time $\left(t_{19}=0.68, p=0.51\right.$; Figure $\left.2 B\right)$. Moreover, a repeated-measures ANOVA with subjective rating type (vividness/confidence) and TMS site (AnG/Vertex) for mean levels of subjective rating did not reveal any significant main effects (rating type: $F_{(1,19)}=1.60, p=0.22$; TMS: $F_{(1,19)}=0.13, p=0.72$ ) nor an interaction $\left(F_{(1,19)}=0.21, p=0.66\right.$; Figure $\left.2 C\right)$. Of central importance, we assessed whether inhibitory rTMS to left AnG modulated the efficiency of subjective ratings during memory retrieval using two robust indices (vivid-d'/d' and meta-d'/d', see Materials and Methods). A repeatedmeasures ANOVA with factors of subjective efficiency type (vividness efficiency/metacognitive efficiency) and TMS site (AnG/vertex) revealed a significant main effect of efficiency type $\left(F_{(1,19)}=69.23, p<0.001\right)$, as well as an interaction $\left(F_{(1,19)}=5.88, p=0.02\right.$; Figure 2D). Follow-up $t$ tests revealed that participants showed significantly lower vividness efficiency following TMS to left $A n G$ compared to vertex $\left(t_{19}=2.96, p<0.01\right)$, whereas no analogous decrement was found in metacognitive efficiency $\left(t_{19}=0.12, p=0.91\right)$. To better characterize the effect of AnG stimulation on vividness, we performed a sign test to verify the extent of changes between TMS to AnG and vertex. Reductions in vividness efficiency were consistent across participants due to TMS to AnG (16/20 reduced; sign test: $p<0.01$; Figure $2 E$ ). Together, these results suggest that the AnG is engaged in the monitoring of vividness and there might be a dissociation between vividness efficiency and confidence efficiency during episodic retrieval. 

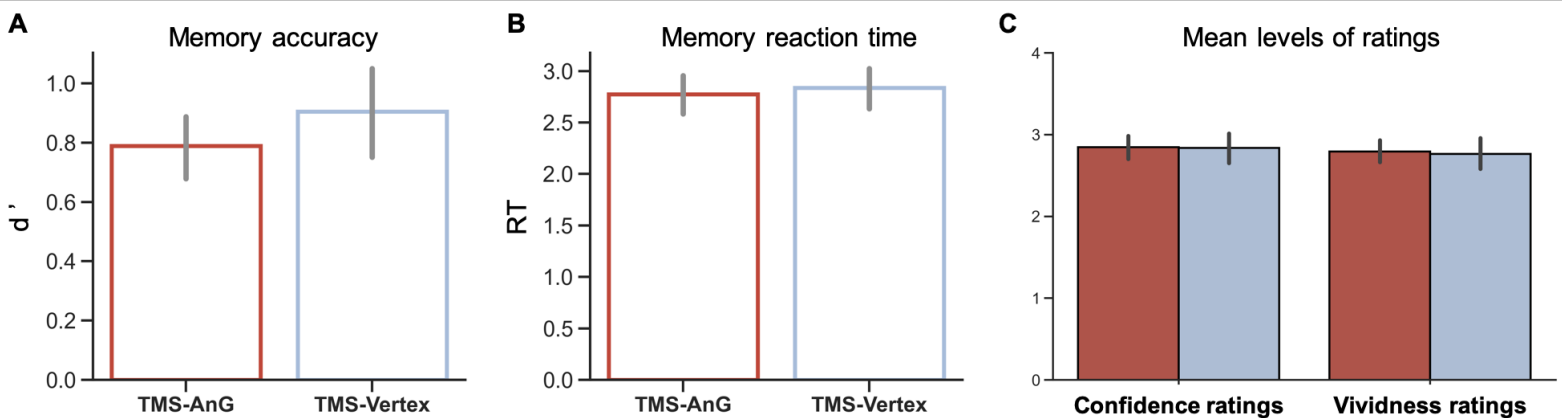

D

E
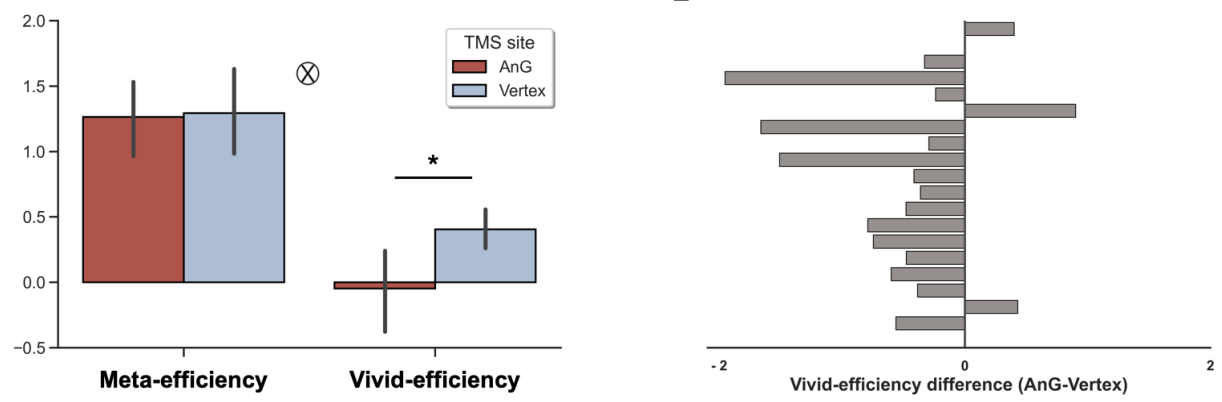

Figure 2. TMS effect on behavioral performance. (A) Accuracy (d') and (B) Reaction times (RTs) in the temporal memory task. (C) Mean levels of confidence ratings and vividness ratings. (D) Metacognitive efficiency and vividness efficiency. (E) Change in vividness efficiency between AnG and vertex stimulation for each participant. Error bars represent SEM. $\otimes$ indicates interaction of subjective reports efficiency by stimulation site in a repeated measures ANOVA. ${ }^{*} p<0.05$

\section{AnG stimulation altered the mediating role of vividness in confidence in the accuracy of memory judgment}

To examine how objective memory accuracy and the two subjective ratings of memory are interrelated in a single statistical framework, we conducted a mediation analysis using objective memory performance as the independent variable and vividness rating as the mediator variable under each TMS condition separately. We hypothesized that the link between objective memory response and confidence might be mediated by the vividness of memory. Under TMS to vertex, vividness ratings were significantly associated with both objective memory performance $(r=0.17$, $p<0.001)$ and confidence ratings $(r=0.34, p<0.001)$. There was also a significant association between objective memory performance and confidence ratings $(r=0.50, p<0.001)$. After adding vividness ratings as a simultaneous predictor, the relationship between objective memory performance and confidence ratings remained intact $(r=0.55, p<0.001)$. The trial-wise mediation analysis revealed that vividness ratings partially mediated the association between objective memory performance and confidence ratings (indirect effect $=0.06, p<0.001,95 \% \mathrm{Cl}=0.04-0.08$; 
Figure 3). By contrast, the vividness ratings did not mediate the relationship between objective memory and confidence ratings following AnG stimulation. The AnG stimulation altered the association between objective memory performance and vividness ratings ( $r=0.05, p=0.149)$, but not confidence ratings $(r=0.52, p<0.001)$. Consistent with our prior results, these findings indicate that, although both vividness ratings and confidence ratings were independently associated with objective memory performance under control site, AnG stimulation selectively impacted the association between vividness ratings and objective memory. Furthermore, these results suggest a mediation between memory performance and confidence through the subjective vividness of memory.

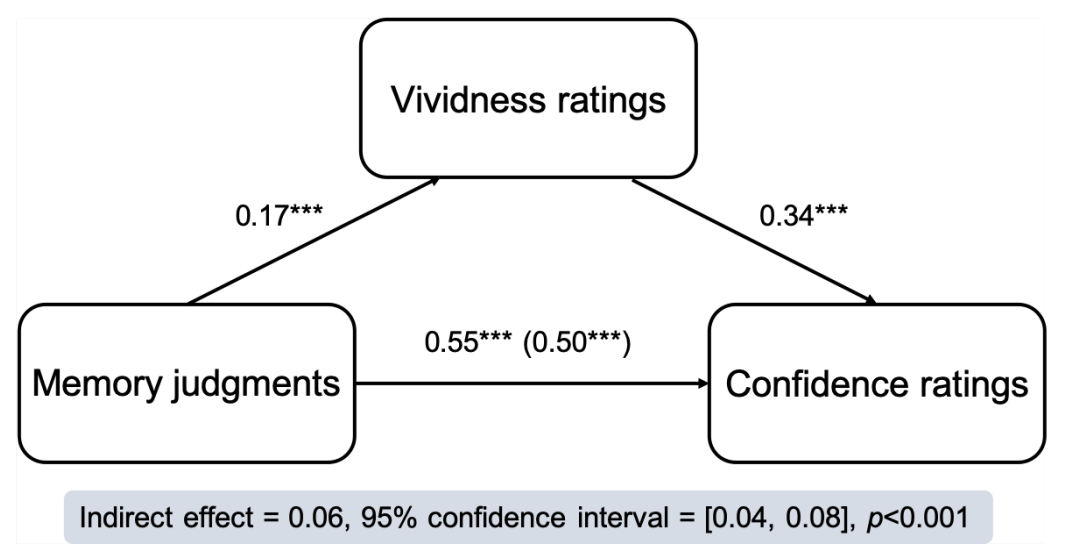

Figure 3. Schematic of mediation analysis. The mediation path diagram shows significant association between memory performance and vividness ratings; vividness ratings and confidence ratings; memory performance and confidence ratings; and a significant mediation effect of vividness on the relationship between memory performance and confidence ratings under TMS to vertex. ${ }^{* * *} \mathrm{p}<0.001$.

Double dissociation between subjective efficiencies manifested in functional connectivity of hippocampus with angular gyrus versus medial anterior prefrontal cortex 
A

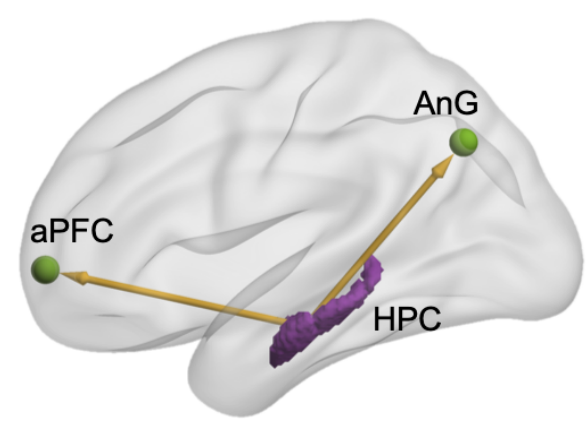

B

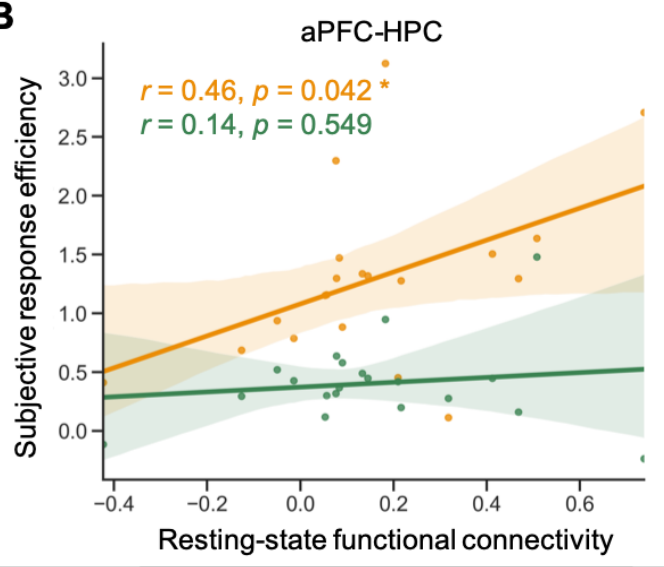

C

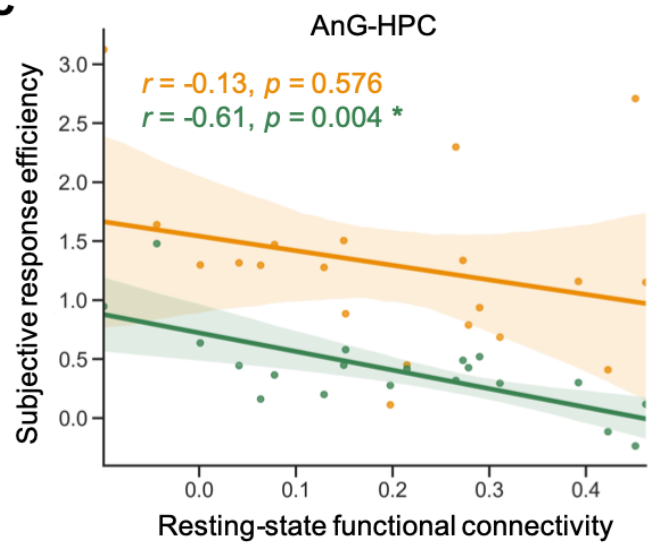

Fig. 4 Resting-state functional connectivity analysis and anatomical double dissociation between the two subjective efficiencies. (A) Display of functional connectivity model of hippocampus (HPC) with angular gyrus (AnG) and medial anterior prefrontal cortex (aPFC). (B) Metacognitive efficiency, but not vividness efficiency, is significantly correlated with aPFC-HPC functional connectivity. (C) Vividness efficiency, but not metacognitive efficiency, is significantly correlated with AnG-HPC functional connectivity.

Having demonstrated that the AnG modulated the efficiency of vividness ratings, we then explored whether the intrinsic functional communication among brain regions was associated with subjective reports efficiencies. Specifically, we examined the relationship between intraindividual variability in subjective report efficiency and the resting-state functional connectivity of the hippocampus (HPC) with angular gyrus (AnG) and medial anterior prefrontal cortex (aPFC). This network has previously been shown to be related to memorial metacognition (Baird et al., 2013). Interestingly, we observed a double dissociation in these functional connections between efficiency of vividness and confidence (Figure 4). The functional connectivity of aPFC-HPC significantly predicted metacognitive efficiency $(r=0.46, p=0.042)$, but not vividness efficiency $(r=0.14, p=0.549$; comparison between correlations: $z=1.801, p=0.036)$. Conversely, the functional connectivity of AnG-HPC was significantly correlated with vividness efficiency ( $r=-0.61$, 
$p=0.004)$, but not metacognitive efficiency $(r=-0.13 p=0.576$; comparison between correlations: $z=1.125, p=0.130$ ), further confirming that the vividness and confidence during memory retrieval may be associated with distinct introspective neural substrates. Moreover, TMS to AnG reduced the correlation between functional connectivity of AnG-HPC and vividness efficiency ( $r=-0.31$, $p=0.189$ ). Interestingly, TMS also altered the association between aPFC-HPC and metacognitive efficiency by flipping the direction ( $r=-0.45, p=0.047)$. Consistent with our prediction, these results revealed that the self-monitoring of vividness and confidence are not only functionally but neurally dissociable.

\section{AnG stimulation eradicated serial dependence effect in both subjective ratings RTs}

We have thus far revealed differential TMS effects on the accuracy of two subjective ratings and their interrelationship with objective memory performance. We next sought to investigate whether the subjective evaluation mechanisms might share similarity in terms of how they incorporate past information into the current decision, or otherwise known as serial dependence effect (Fischer and Whitney, 2014; Rahnev et al., 2020, 2015). Given that RT is a defining element of the tradeoff between speed and accuracy that characterizes decisions, the presence of serial dependence on RT can provide important insights into the nature of subjective awareness generation. To test for serial dependence in vividness RTs and confidence RTs separately, we performed a series of mixed regression analyses predicting subjective rating RTs with fixed effects for the recent trial history up to seven trials back and random intercepts for each participant. We also explicitly tested for any differing involvement of AnG in generating subjective estimation during memory retrieval. We found that there was autocorrelation in vividness RTs up to lag-3 (all p-values< 0.05 ; Figure $5 A$ ) under TMS to vertex. Following TMS to AnG, such serial dependence was not found in vividness RTs. Furthermore, we also observed autocorrelation in confidence RTs up to lag-2 (all p-values < 0.05; Figure 5B) under TMS control condition and such serial dependence effect was also reduced by AnG stimulation. These results replicated the existence of serial dependence in confidence RT and revealed serial dependence in vividness rating RTs, and that both are modulated by AnG stimulation. The findings of such serial spill-over bias in both subjective estimations and their susceptibility to AnG stimulation suggested their similarity in terms of subjective experience generations during memory retrieval. 

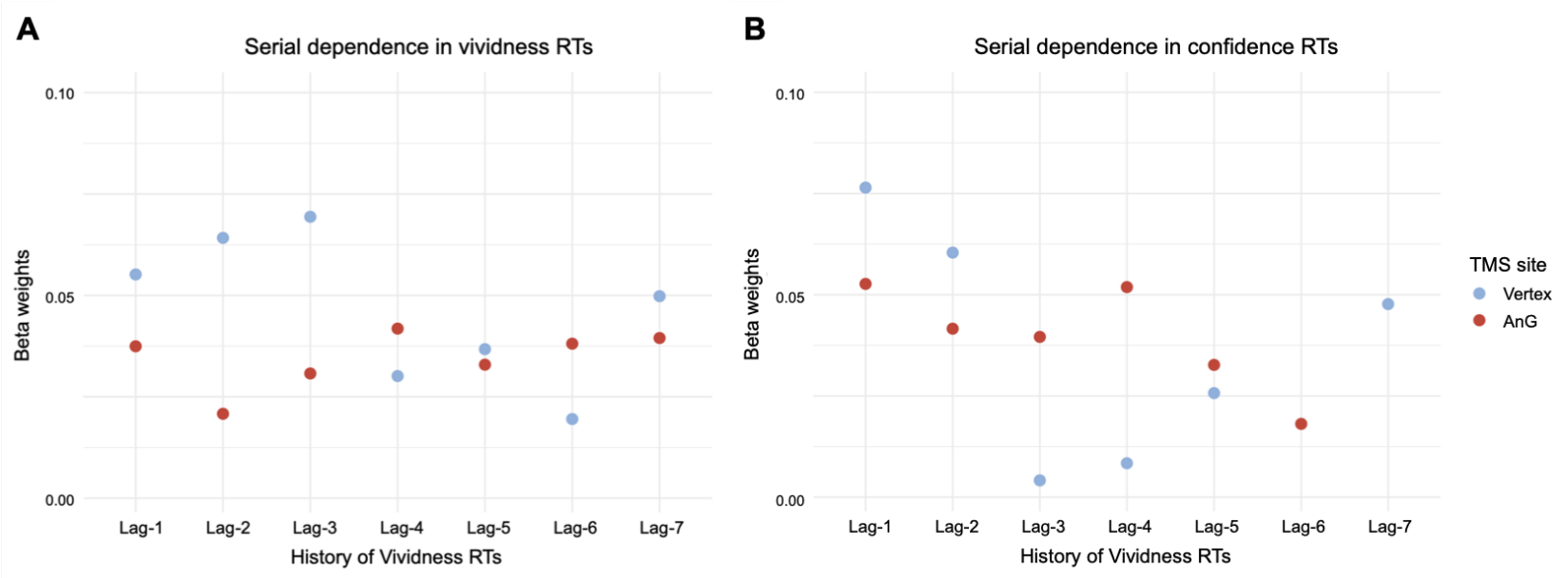

Figure 5. Serial dependence in subjective reports RTs. (A) Autocorrelation in vividness RTs was observed up to lag-3 under TMS to vertex (all p-values < 0.05; blue dots). No reliable autocorrelation was found in vividness RTs after TMS to AnG (red dots). (B) Autocorrelation was found in confidence RTs up to lag-2 under TMS to vertex (all p-values < 0.05). Such autocorrelation in confidence RT was not found after TMS to AnG.

In sum, consistent with our predictions, these findings establish a specific role of AnG and its mediating effects as well as its functional connection with the hippocampus in subserving our perceived vividness of memory retrieval. The direct comparison with the metacognitive counterpart (indexed by confidence ratings) suggested functional and anatomical dissociation between the two subjective efficiencies in these mnemonic processes.

\section{Discussion}

How do we obtain accurate access to our memory? Much of what we know about subjective aspects of memory comes from experimental work measuring the relationship between the level of confidence or vividness rating and brain region during memory retrieval. Yet the ability to accurately monitor internal subjective mnemonic experience has remained poorly understood. Here, across domains of memory and metacognition, we explore the question of what information is being used for confidence rating in memory judgement. Specifically, we raise the question whether subjective confidence and vividness of memory reflect distinct introspective capacities. By administering non-invasive pre-retrieval stimulation to the angular gyrus, a candidate region supporting the subjective components of memory, we provide evidence for a causal role for angular gyrus specifically in vividness efficiency. Critically, we show novel behavioral and functional MRI evidence that the ability of monitoring vividness of memory is indeed functionally and anatomically dissociable from confidence, echoing the notion that confidence is beyond the quality of sensory evidence. 
One of the innovative aspects of this work is that we isolate the processes underlying judgments of vividness and confidence during episodic memory retrieval. We observed that temporary disruption of the angular gyrus led to difference in the quality of vividness ratings while leaving the confidence ratings impact, suggesting that vividness and confidence of memory are two separable subjective experiences. These results are compatible with prior findings that the angular gyrus is involved in the subjective experience of remembering (Kuhl and Chun, 2014; Yazar et al., 2014) but not in confidence-related metacognition. One possibility is that vividness of memory reflects something akin to the perception of past events, analogous to the "attention to memory" (AtoM) account (Cabeza, 2008; Ciaramelli et al., 2008; Hutchinson et al., 2009). Retrieval from long-term memory demands selection between specific memories competing for recall (Badre et al., 2005). Previous theories have advanced the analogies between selection in the perceptual domain and selection during memory retrieval (Cabeza, 2008; Wagner et al., 2005). Accordingly, the AtoM account proposes that the parietal mechanisms (including angular gyrus) support goal-directed attention toward the maintenance of mnemonic cues as well as facilitate the monitoring of episodic memory retrieval (Kwok and Macaluso, 2015; Hutchinson et al., 2009). In light of the view, the subjective sensed vividness during memory recall may thus represent a product of internal attentional processes rather than a subjective evaluation of memory quality, such as confidence. It is then plausible that the TMS to the angular gyrus disrupts the shifting and allocation of attention to internal representations, resulting in less accurate perceived vividness of memory. A potential future direction following this work is to examine the degree of anatomical and functional convergence between the vividness rating and reflective attention.

Although prior work has shown consistent involvement of angular gyrus in recollection vividness, the contribution of angular gyrus to the self-monitoring of this subjective aspect of memory has yet to be directly tested. Previous studies have linked activity in angular gyrus with rated vividness (Bonnici et al., 2016; Kuhl and Chun, 2014) and reported that patients with lateral parietal lesions show diminished vividness of their memories (Hower et al., 2014; Simons et al., 2010; Berryhill et al., 2007). However, we did not observe any TMS effect on the overall rated vividness. Rather, instead of crudely using the reported vividness, here we applied the concept of using performance and confidence correspondence (a quantitative measure of metacognition) to derive the degree of correspondence between rated vividness and objective memory accuracy. This approach enables us to estimate the TMS effect on the vividness efficiency independently from the level of vividness and objective memory performance. Moreover, we asked participants to rate the vividness of the mental replay before any mnemonic decision, which allows for an uncontaminated assessment of the richness of mental experience prior to any memory judgement (Siedlecka et al., 2016). Our findings add to this limited literature by demonstrating a causal role for the angular gyrus in vividness efficiency. One interpretation of these results is that the angular gyrus may act 
as an accumulator in service of mnemonic decisions (Wagner et al., 2005). It has been previously proposed that memory retrieval is accomplished by a diffusion process during which evidence for a memory decision is accumulated (Ratcliff, 1978), and the parietal cortex (including angular gyrus) is thought to play a role in the integration of sensory information (Gold and Shadlen, 2007; Shadlen and Newsome, 2001). This hypothesis is compatible with our data, accommodating the finding that TMS to angular gyrus affected the correspondence between vividness and memory performance, but neither the mean level of rated vividness and objective memory performance. More broadly, our findings clarify a role for angular gyrus to accurately gauging the vividness of memory and further support the notion that angular gyrus participates in accumulating and integrating information in support of mnemonic processes.

Intrinsic individual differences in functional connectivity between brain structures have informed our understanding of the varied ability to introspect about self-performance (Baird et al., 2013; Fleming et al., 2010; Ye et al., 2019). Here we found that resting-state functional connectivity of hippocampus and angular gyrus is specifically associated with vividness efficiency, while the connectivity of hippocampus and medial aPFC predicts metacognitive efficiency across individuals. First, we were able to replicate an analysis by (Baird et al., 2013), who showed that the ability to make accurate metacognitive of memory was associated with increased connectivity between medial aPFC and hippocampus. Second, this double dissociation observed in functional connectivity of hippocampus with angular gyrus versus medial aPFC is in line with our behavioral results that vividness and confidence may depend on dissociable neural substrates. Collectively, these results provide direct and unique support for the differentiation account of subjective assessments of memory by functionally and anatomically dissociating the monitoring of vividness from confidence.

In the literature on perceptual metacognition, theories of confidence generation posit that the central processing of evidence leading to a perceptual decision also establishes a level of confidence (Fetsch et al., 2014; Sanders et al., 2016). Some argue that confidence rating is additionally corrupted by a meta-level noise (Shekhar and Rahnev, 2018; De Martino et al., 2012). In contrast, it remains less studied for the origins of confidence in the context of episodic memory decision making. Here, in an elucidation of the relationship between vividness, confidence, and objective memory performance, we found that vividness mediates the association between confidence and objective performance. This indicates that the sensed vividness of memory is instrumentally used for the computation of confidence. Consideration of the relative contribution of subjective feeling of vividness in generating confidence, especially for more naturalistic paradigms involving continuous streams of multisensory information and mnemonic experiences, is thus paramount. Although the issue of deriving the best model for memory confidence is not our focus here, we hope that our findings provide some new insights into the confidence generation in episodic memory decision for future work. A critical avenue for future studies is to 
exploit what other information beyond subjective vividness is being used for confidence generation in episodic memory.

In closing, we demonstrate the contribution of angular gyrus to vividness processing in terms of its mediating effect, its regional (by TMS) and cross-regional connectivity characteristics (by resting-state $\mathrm{MRI}$ ). These findings suggest future investigation of conscious mnemonic experiences would benefit from taking memory vividness, their computation, and its anatomical profile into consideration.

\section{Materials and Methods}

\section{Participants}

Twenty healthy young adults took part in this study (11 females and 9 males, mean age $=22.70$ years, $S D=2.8$, range $=18-26$ ). The sample size was determined a priori based on prior TMS studies using within-subjects design and employing similar analysis techniques to those used in the current study (Ye et al., 2018). All participants were right-handed with normal or corrected-tonormal vision, and had no contraindications for MRI or TMS. Each of them participated in two experimental sessions, giving us a within-subjects comparison to assess the influence of TMS to angular gyrus on memory. Data from three additional participants were excluded from data analyses: one participant did not complete the experiment due to anxiety and the other two inadvertently hit the wrong response key throughout a whole test session. Participants were recruited from the East China Normal University undergraduate and graduate student population and compensated for their participation. The East China Normal University Committee on Human Research Protection approved the experimental protocol and all participants gave their written informed consent. All participants self-reported to be native Chinese speakers and had not previously seen any episodes of Black Mirror.

\section{Procedure}

Participants completed a baseline session and two experimental sessions on separate days in a within-subjects design (Figure 1A). Following standard MRI and TMS safety screening, participants first underwent a baseline session where structural MRI scans and resting-state fMRI scans were obtained. The structural MRI scans were used to define the subjective-specific stimulation locations and enable accurate navigation. Each experimental session consisted of two phases separated by one day: an approximately 1-hr encoding session, during which participants watched one Black Mirror movie, and a retrieval session one day later, during which participants received either rTMS over the left-AnG or over the vertex and completed a memory retrieval test. 
The retrieval began immediately after rTMS and lasted $50 \mathrm{~min}$. In the retrieval phase, participants recalled relevant scenarios based on a cue image, rated their subjective vividness of the mental replay, made temporal memory judgments, and rated their confidence of the memory judgments (Figure 1C).

\section{Stimuli}

Participants viewed two episodes of the British television series Black Mirror (Figure 1B; the first episode of Season 3, Nosedive, and the third episode of Season 3, Shut up and Dance) with Chinese dubbing. Each episode was assigned to one of the experimental sessions. Nosedive was 58 min long and Shut up and Dance was 52 min long. For the subsequent memory retrieval test, 180 triads of still frames were extracted from each movie based on the following criteria: i) for each triad, one cue frame and two still images for temporal memory judgments were from the adjacent scenes; ii) the absolute temporal distance between cue frame and temporally closer one to the cue was fixed. To further increase task difficulty, we selected the stimuli from four difficulty settings: hard/easy with left/right target (Figure 1C). The occurrence of event boundaries was identified using subjective annotations. Two external observers, who did not take part in the experimental sessions of the current study and had no knowledge of the experimental design, viewed each of the movies and annotated with precision the temporal point at which they felt "a new event is starting; these are points in the movie when there is a major change in topic, location or time." Participants were also asked to write down a short title for each event. With the participants' boundary annotations, we looked for those boundary time points that were consistent across observers. This resulted in 50 scenes in Nosedive and 43 scenes in Shut up and Dance.

\section{Memory test}

In the memory test, participants were first presented with an image cue abstracted from the movie and asked to mentally recall related scenarios in the movie as detailed as possible for $6 \mathrm{~s}$. Following the mental replay, participants were allowed $3 s$ to rate their vividness of the memory by selecting a number from 1 to 4 ("not vivid" to "very vivid"). After the vividness rating, participants were presented with another two still frames from the movie and were asked to choose which of the two frames was temporally closer to the cue frame in the movie. On each trial, the stimulus presentation and response window lasted for $5 \mathrm{~s}$. Each temporal memory judgment was followed by a subjective confidence rating of their choice on a scale from 1 to 4 ("not confident" to "very confident"). $3 \mathrm{~s}$ were allowed for confidence ratings. There were two sets of temporal memory judgment and confidence rating following each cued recall. No feedback was provided during the memory test. 


\section{MRI data acquisition}

Participants were scanned in a 3-tesla Siemens Trio magnetic resonance imaging scanner with a 64-channel head coil. Structural MRI images were obtained using a T1-weighted (T1w) multiecho MPRAGE protocol (field of view $=224 \mathrm{~mm}$, TR $=2300 \mathrm{~ms}$, TE $=2.25 \mathrm{~ms}$, flip angle = $8^{\circ}$, voxel size $=1 \times 1 \times 1 \mathrm{~mm}, 192$ sagittal slices) to stereotaxically guide the stimulation.

\section{Repetitive transcranial magnetic stimulation (rTMS)}

In each experimental session, participants received rTMS to either the left AnG or vertex before the memory test. The stimulation site order was counterbalanced across participants. rTMS was applied using a Magstim Rapid2 magnetic stimulator connected to a $70 \mathrm{~mm}$ double air film coil. The structural data obtained from each participant were used in Brainsight 2.0, a computerized frameless stereotaxic system (Rogue Research), to identify the target brain regions on a subjectby-subject basis. The stimulation sites were selected in the system by transformation of the Montreal Neurological Institute (MNI) stereotaxic coordinates to participant's normalized brain. The sites stimulated were located in the left AnG at the MNI coordinate $x=-43, y=-66, z=38$ (Vilberg and Rugg, 2008), and in a control area on the vertex, which was identified at the point of the same distance to the left and the right pre-auricular, and of the same distance to the nasion and the inion (Figure 1D). To target the selected stimulation sites, four fiducial points located on the face were used to co-register the anatomical MRI to the participant's head using an infrared pointer. The real-time locations of the TMS coil and the participant's head were monitored by an infrared camera using a Polaris Optical Tracking System (Northern Digital).

rTMS was applied at $1 \mathrm{~Hz}$ frequency for a continuous duration of $20 \mathrm{~min}$ (1200 pulses in total) at $110 \%$ of active motor threshold (MT), which was defined as the lowest TMS intensity delivered over the motor cortex necessary to elicit visible twitches of the right index finger in at least 5 of 10 consecutive pulses (Rossini et al., 2015). During stimulation, participants wore earplugs to attenuate the sound of the stimulating coil discharge. The coil was held to the scalp of the participant with a custom coil holder and the participant's head was propped in a comfortable position. This particular stimulation magnitude and protocols of rTMS is known to induce efficacious intracortical inhibitory effects for over 60 min (Rossini et al., 2015; Thut and PascualLeone, 2010). Given that our task lasted $50 \mathrm{~min}$, the TMS effects should have been long-lasting enough for the task. Although these inhibitory effects are known to level off within hours by the end of the stimulation, for safety reasons and to avoid carryover effects of rTMS across sessions, experimental session 1 and 2 were conducted on separate days with at least 3 days apart. 


\section{Quantification of subjective memory performance}

Metacognition refers to one's subjective access to their own cognitive processes, and is computed by estimating how accurate subjective ratings distinguish between correct and incorrect responses. For completeness and comparability with previous metacognition work, we estimated memory metacognitive ability using the confidence ratings. To assess whether participants' confidence ratings were reliably related to their objective memory performance, we computed meta-d', a metric that quantifies the metacognitive sensitivity and is independent of confidence bias, using a Bayesian model-based method (Fleming, 2017; Fleming and Lau, 2014). Given the metric, meta-d', is expressed in the same units as d', it allows a direct comparison between objective performance and metacognitive sensitivity. We therefore calculated metacognitive efficiency using the ratio meta-d'/d', which indexes participant's metacognitive sensitivity irrespective of objective performance. To quantify the extent to which participants' subjective vividness ratings tracked their objective memory performance, we applied the same hierarchical Bayesian framework of metacognitive efficiency to vividness ratings and computed a metric termed vividness efficiency (vivid-d'/d').

\section{Resting-state functional connectivity analysis}

For connectivity analysis of resting-state data, resting-state functional data were first converted to Brain Imaging Data Structure (BIDS) format and verified using the BIDS validator. Data preprocessing was performed using fMRIPrep (Esteban et al., 2019) with the default processing steps, including skull stripping, motion correction, brain tissue segmentation, slice time correction, and co-registration and affine transformation of the functional volumes to corresponding T1 $\mathrm{w}$ and subsequently to $\mathrm{MNI}$ space. For further details of the pipeline, please refer to the online documentation: https://fmriprep.org/.

To estimate connectivity, we defined 3 a prior regions of interest (ROIs), including AnG, medial aPFC and hippocampus. We included the AnG seed region as a sphere of $6 \mathrm{~mm}$ diameter with centers at the stimulation site ( $x=-43, y=66, z=38$,(Vilberg and Rugg, 2008)). The medial aPFC $\mathrm{ROI}$ was defined as a sphere of $6 \mathrm{~mm}$ diameter with centers at $6,58,0$. The location of the medial aPFC was based on a region described in a meta-analysis (Gilbert et al., 2006), which has been previously reported to be important to memorial metacognition (Baird et al., 2013). The hippocampal ROI was obtained from a medial temporal lobe atlas (Ritchey et al., 2015). ROI-ROI resting-state functional connectivity analyses were performed using the CONN toolbox (WhitfieldGabrieli and Nieto-Castanon, 2012). Preprocessed functional data were first linearly detrended and a commonly used bandpass filter $(0.008-0.09 \mathrm{~Hz})$ was applied to isolate low-frequency 
fluctuations characteristic of resting-state fMRI and attenuate signals outside of that range. White matter and CSF confounds were removed using the aCompCor method. To ensure no voxels were included in mean estimates from outside ROls, we performed all analyses using unsmoothed functional data.

\section{Acknowledgements}

This research received support from Science and Technology Commission of Shanghai Municipality (Grant No. 201409002800), the National Natural Science Foundation of China (Grant No. 32071060), and Joint Research Institute for Science and Society Incubating Project (S.C.K.).

\section{References}

Allen M, Frank D, Schwarzkopf DS, Fardo F, Winston JS, Hauser TU, Rees G. 2016. Unexpected arousal modulates the influence of sensory noise on confidence. eLife 5:e18103. doi:10.7554/eLife.18103

Allen M, Glen JC, Müllensiefen D, Schwarzkopf DS, Fardo F, Frank D, Callaghan MF, Rees G. 2017. Metacognitive ability correlates with hippocampal and prefrontal microstructure. Neurolmage 149:415-423. doi:10.1016/j.neuroimage.2017.02.008

Badre D, Poldrack RA, Paré-Blagoev EJ, Insler RZ, Wagner AD. 2005. Dissociable Controlled Retrieval and Generalized Selection Mechanisms in Ventrolateral Prefrontal Cortex. Neuron 47:907-918. doi:10.1016/j.neuron.2005.07.023

Baird B, Smallwood J, Gorgolewski KJ, Margulies DS. 2013. Medial and Lateral Networks in Anterior Prefrontal Cortex Support Metacognitive Ability for Memory and Perception. $J$ Neurosci 33:16657 LP - 16665.

Bang J, Shekhar M, Rahnev D. 2019. Sensory Noise Increases Metacognitive Efficiency. J Exp Psychol Gen 148:437-452. doi:10.1037/xge0000511

Berryhill ME, Phuong L, Picasso L, Cabeza R, Olson IR. 2007. Parietal Lobe and Episodic Memory: Bilateral Damage Causes Impaired Free Recall of Autobiographical Memory. J Neurosci 27:14415-14423. doi:10.1523/JNEUROSCI.4163-07.2007

Bonnici HM, Richter FR, Yazar Y, Simons JS. 2016. Multimodal Feature Integration in the Angular Gyrus during Episodic and Semantic Retrieval. J Neurosci Off J Soc Neurosci 36:5462-71. doi:10.1523/JNEUROSCI.4310-15.2016

Cabeza R. 2008. Role of parietal regions in episodic memory retrieval: The dual attentional processes hypothesis. Neuropsychologia, Part Special Issue: What is the Parietal Lobe Contribution to Human Memory? 46:1813-1827. doi:10.1016/j.neuropsychologia.2008.03.019

Chua EF, Schacter DL, Sperling RA. 2009. Neural Correlates of Metamemory: A Comparison of Feeling-of-Knowing and Retrospective Confidence Judgments. J Cogn Neurosci 21:1751-1765. doi:10.1162/jocn.2009.21123

Ciaramelli E, Grady CL, Moscovitch M. 2008. Top-down and bottom-up attention to memory: A hypothesis (AtoM) on the role of the posterior parietal cortex in memory retrieval. Neuropsychologia, Part Special Issue: What is the Parietal Lobe Contribution to Human Memory? 46:1828-1851. doi:10.1016/j.neuropsychologia.2008.03.022 
De Martino B, Fleming SM, Garrett N, Dolan RJ. 2012. Confidence in value-based choice. Nat Neurosci 16:105-110. doi:10.1038/nn.3279

Esteban O, Markiewicz CJ, Blair RW, Moodie CA, Isik AI, Erramuzpe A, Kent JD, Goncalves M, DuPre E, Snyder M, Oya H, Ghosh SS, Wright J, Durnez J, Poldrack RA, Gorgolewski KJ. 2019. fMRIPrep: a robust preprocessing pipeline for functional MRI. Nat Methods 16:111-116. doi:10.1038/s41592-018-0235-4

Fetsch CR, Kiani R, Newsome WT, Shadlen MN. 2014. Effects of Cortical Microstimulation on Confidence in a Perceptual Decision. Neuron 83:797-804. doi:10.1016/j.neuron.2014.07.011

Fischer J, Whitney D. 2014. Serial dependence in visual perception. Nat Neurosci 17:738-743. doi:10.1038/nn.3689

Fleming SM. 2017. HMeta-d: hierarchical Bayesian estimation of metacognitive efficiency from confidence ratings. Neurosci Conscious 3:1-14. doi:10.1093/nc/nix007

Fleming SM, Daw ND. 2016. Self-evaluation of decision performance: A general Bayesian framework for metacognitive computation. Psychol Rev 124:1-59. doi:10.1002/smll.))

Fleming SM, Lau H. 2014. How to measure metacognition. Front Hum Neurosci 8:1-9. doi:10.3389/fnhum.2014.00443

Fleming SM, Weil RS, Nagy Z, Dolan RJ, Rees G. 2010. Relating Introspective Accuracy to Individual Differences in Brain Structure. Science 329:1541-1543. doi:10.1126/science.1191883

Ford JH, Kensinger EA. 2016. Effects of internal and external vividness on hippocampal connectivity during memory retrieval. Neurobiol Learn Mem, Hippocampal Interactions with Brain Networks that Influence Learning \& Memory 134:78-90. doi:10.1016/j.nlm.2015.12.007

Gilbert SJ, Spengler S, Simons JS, Steele JD, Lawrie SM, Frith CD, Burgess PW. 2006. Functional Specialization within Rostral Prefrontal Cortex (Area 10): A Meta-analysis. J Cogn Neurosci 18:932-948. doi:10.1162/jocn.2006.18.6.932

Gilboa A, Winocur G, Grady CL, Hevenor SJ, Moscovitch M. 2004. Remembering Our Past: Functional Neuroanatomy of Recollection of Recent and Very Remote Personal Events. Cereb Cortex 14:1214-1225. doi:10.1093/cercor/bhh082

Gold JI, Shadlen MN. 2007. The Neural Basis of Decision Making. Annu Rev Neurosci 30:535574. doi:10.1146/annurev.neuro.29.051605.113038

Hower KH, Wixted J, Berryhill ME, Olson IR. 2014. Impaired perception of mnemonic oldness, but not mnemonic newness, after parietal lobe damage. Neuropsychologia 56:409-417. doi:10.1016/J.NEUROPSYCHOLOGIA.2014.02.014

Humphreys GF, Ralph MAL, Simons JS. 2021. A Unifying Account of Angular Gyrus Contributions to Episodic and Semantic Cognition. Trends Neurosci 0. doi:10.1016/j.tins.2021.01.006

Hutchinson JB, Uncapher MR, Wagner AD. 2009. Posterior parietal cortex and episodic retrieval: Convergent and divergent effects of attention and memory. Learn Mem 16:343-356. doi:10.1101/lm.919109

Janet Metcalfe. 1997. Metacognition: Knowing about knowing. Boston MIT 102:519-519. doi:10.1016/S0013-4694(97)84006-4

Kuhl BA, Chun MM. 2014. Successful remembering elicits event-specific activity patterns in lateral parietal cortex. J Neurosci Off J Soc Neurosci 34:8051-60. doi:10.1523/JNEUROSCI.4328-13.2014

Kwok SC, Macaluso E. 2015. Exogenous features versus prior experiences modulate different subregions of the right IPL during episodic memory retrieval. Sci Rep 5:11248. doi:10.1038/srep11248

Maniscalco B, Lau H. 2016. The signal processing architecture underlying subjective reports of sensory awareness. Neurosci Conscious 2016. doi:10.1093/nc/niw002 
Maniscalco B, Lau H. 2012. A signal detection theoretic approach for estimating metacognitive sensitivity from confidence ratings. Conscious Cogn 21:422-430. doi:10.1016/j.concog.2011.09.021

Morales J, Lau H, Fleming SM. 2018. Domain-General and Domain-Specific Patterns of Activity Supporting Metacognition in Human Prefrontal Cortex. J Neurosci Off J Soc Neurosci 38:3534-3546. doi:10.1523/JNEUROSCI.2360-17.2018

Qin S, Marle HJF van, Hermans EJ, Fernández G. 2011. Subjective Sense of Memory Strength and the Objective Amount of Information Accurately Remembered Are Related to Distinct Neural Correlates at Encoding. J Neurosci 31:8920-8927. doi:10.1523/JNEUROSCI.2587-10.2011

Rahnev D, Desender K, Lee ALF, Adler WT, Aguilar-Lleyda D, Akdoğan B, Arbuzova P, Atlas LY, Balcı F, Bang JW, Bègue I, Birney DP, Brady TF, Calder-Travis J, Chetverikov A, Clark TK, Davranche K, Denison RN, Dildine TC, Double KS, Duyan YA, Faivre N, Fallow K, Filevich E, Gajdos T, Gallagher RM, de Gardelle V, Gherman S, Haddara N, Hainguerlot M, Hsu T-Y, Hu X, Iturrate I, Jaquiery M, Kantner J, Koculak M, Konishi M, Koß C, Kvam PD, Kwok SC, Lebreton M, Lempert KM, Ming Lo C, Luo L, Maniscalco B, Martin A, Massoni S, Matthews J, Mazancieux A, Merfeld DM, O'Hora D, Palser ER, Paulewicz B, Pereira M, Peters C, Philiastides MG, Pfuhl G, Prieto F, Rausch M, Recht S, Reyes G, Rouault M, Sackur J, Sadeghi S, Samaha J, Seow TXF, Shekhar M, Sherman MT, Siedlecka M, Skóra Z, Song C, Soto D, Sun S, van Boxtel JJA, Wang S, Weidemann CT, Weindel G, Wierzchoń M, Xu X, Ye Q, Yeon J, Zou F, Zylberberg A. 2020. The Confidence Database. Nat Hum Behav 4:317-325. doi:10.1038/s41562-0190813-1

Rahnev D, Koizumi A, McCurdy LY, D’Esposito M, Lau H. 2015. Confidence Leak in Perceptual Decision Making. Psychol Sci 26:1664-1680. doi:10.1177/0956797615595037

Ratcliff R. 1978. A theory of memory retrieval. Psychol Rev 85:59-108. doi:10.1037/0033295X.85.2.59

Ritchey M, Montchal ME, Yonelinas AP, Ranganath C. 2015. Delay-dependent contributions of medial temporal lobe regions to episodic memory retrieval. eLife 4:e05025. doi:10.7554/eLife.05025

Rossini PM, Burke D, Chen R, Cohen LG, Daskalakis Z, Di lorio R, Di Lazzaro V, Ferreri F, Fitzgerald PB, George MS, Hallett M, Lefaucheur JP, Langguth B, Matsumoto $H$, Miniussi C, Nitsche MA, Pascual-Leone A, Paulus W, Rossi S, Rothwell JC, Siebner HR, Ugawa Y, Walsh V, Ziemann U. 2015. Non-invasive electrical and magnetic stimulation of the brain, spinal cord, roots and peripheral nerves: Basic principles and procedures for routine clinical and research application. An updated report from an I.F.C.N. Committee. Clin Neurophysiol 126:1071-1107. doi:10.1016/j.clinph.2015.02.001

Sanders JI, Hangya B, Kepecs A. 2016. Signatures of a Statistical Computation in the Human Sense of Confidence. Neuron 90:499-506. doi:10.1016/j.neuron.2016.03.025

Shadlen MN, Newsome WT. 2001. Neural Basis of a Perceptual Decision in the Parietal Cortex (Area LIP) of the Rhesus Monkey. J Neurophysiol 86:1916-1936. doi:10.1152/jn.2001.86.4.1916

Shekhar M, Rahnev D. 2018. Distinguishing the roles of dorsolateral and anterior PFC in visual metacognition. J Neurosci Off J Soc Neurosci 3484-17. doi:10.1523/JNEUROSCI.348417.2018

Siedlecka M, Paulewicz B, Wierzchoń M. 2016. But I Was So Sure! Metacognitive Judgments Are Less Accurate Given Prospectively than Retrospectively. Front Psychol 7. doi:10.3389/fpsyg.2016.00218

Simons JS, Peers PV, Mazuz YS, Berryhill ME, Olson IR. 2010. Dissociation between memory accuracy and memory confidence following bilateral parietal lesions. Cereb Cortex $N Y$ N 1991 20:479-85. doi:10.1093/cercor/bhp116 
Thut G, Pascual-Leone A. 2010. A Review of Combined TMS-EEG Studies to Characterize Lasting Effects of Repetitive TMS and Assess Their Usefulness in Cognitive and Clinical Neuroscience. Brain Topogr 22:219-232. doi:10.1007/s10548-009-0115-4

Tulving E. 1985. Memory and consciousness. Can Psychol Can 26:1-12. doi:10.1037/h0080017

Tulving E. 1972. Episodic and semantic memoryOrganization of Memory. Oxford, England: Academic Press. pp. xiii, 423-xiii, 423.

Vilberg KL, Rugg MD. 2008. Memory retrieval and the parietal cortex: A review of evidence from a dual-process perspective. Neuropsychologia, Part Special Issue: What is the Parietal Lobe Contribution to Human Memory? 46:1787-1799. doi:10.1016/j.neuropsychologia.2008.01.004

Wagner AD, Shannon BJ, Kahn I, Buckner RL. 2005. Parietal lobe contributions to episodic memory retrieval. Trends Cogn Sci 9:445-453. doi:10.1016/j.tics.2005.07.001

Whitfield-Gabrieli S, Nieto-Castanon A. 2012. Conn: A Functional Connectivity Toolbox for Correlated and Anticorrelated Brain Networks. Brain Connect 2:125-141. doi:10.1089/brain.2012.0073

Wynn SC, Hendriks MPH, Daselaar SM, Kessels RPC, Schutter DJLG. 2018. The posterior parietal cortex and subjectively perceived confidence during memory retrieval. Learn Mem 382-389. doi:10.1101/lm.048033.118.25

Yazar Y, Bergström ZM, Simons JS. 2014. Continuous Theta Burst Stimulation of Angular Gyrus Reduces Subjective Recollection. PLOS ONE 9:e110414. doi:10.1371/journal.pone.0110414

Ye Q, Zou F, Dayan M, Lau H, Hu Y, Kwok SC. 2019. Individual susceptibility to TMS affirms the precuneal role in meta-memory upon recollection. Brain Struct Funct. doi:10.1007/s00429-019-01909-6

Ye Q, Zou F, Lau H, Hu Y, Kwok SC. 2018. Causal Evidence for Mnemonic Metacognition in Human Precuneus. J Neurosci Off J Soc Neurosci 38:6379-6387. doi:10.1523/JNEUROSCI.0660-18.2018 